\title{
Path Integral Quantization of Nonconservative Systems
}

\author{
Ola A. Jarab'ah ${ }^{*}$, Khaled I. Nawafleh ${ }^{2}$ \\ ${ }^{1}$ Applied Physics Department, Faculty of Science, Tafila Technical University, Tafila, Jordan \\ ${ }^{2}$ Department of physics, Faculty of Science, Mu’tah University, Al-Karak, Jordan \\ Email: *oasj85@yahoo.com
}

How to cite this paper: Jarab'ah, O.A. and Nawafleh, K.I. (2018) Path Integral Quantization of Nonconservative Systems. Journal of Applied Mathematics and Physics, 6, 1637-1641.

https://doi.org/10.4236/jamp.2018.68139

Received: July 8, 2018

Accepted: August 7, 2018

Published: August 10, 2018

Copyright ( $) 2018$ by authors and Scientific Research Publishing Inc. This work is licensed under the Creative Commons Attribution International License (CC BY 4.0).

http://creativecommons.org/licenses/by/4.0/

\begin{abstract}
In this paper nonconservative systems are investigated within the framework of Euler Lagrange equations. The solutions of these equations are used to find the principal function $\mathbf{S}$, this function is used to formulate the wave function and then to quantize these systems using path integral method. One example is considered to demonstrate the application of our formalism.
\end{abstract}

\section{Keywords}

Path Integral, Irregular Lagrangian, Nonconservative Systems, Wave Function, Principal Function

\section{Introduction}

The path integral formulation of quantum mechanics is a description of quantum theory that generalizes the action principle of classical mechanics. It replaces the classical notion of a single, unique classical trajectory for a system with a sum, or functional integral over the canonical phase space. The basic idea of the path integral formulation can be traced back by [1] who introduced the Wiener integral for solving problems in diffusion and Brownian motion. This idea was extended to the use of the Lagrangian in quantum mechanics [2]. The complete method was developed by [3].

The canonical formalism for investigating singular systems was developed by [4] [5] [6]. In this formalism, the equations of motion are obtained as total differential equations. Depending on this method, the path integral quantization of constrained Lagrangian systems has been investigated by [7] [8] [9] [10]. Moreover, the quantization of constrained systems has been studied using the WKB approximation [11] [12] [13]. Recently, the quantization of dissipative systems has been studied using the WKB approximation and path integral method which 
depending on Hamiltonian mechanics [14] [15]. More recently, regular Lagrangian is quantized using path integral method in [16].

This paper is mainly concerned with nonconservative systems which are characterized by irregular Lagrangian using canonical approach and the quantization of these systems using the canonical path integral method.

Our present work is organized as follows. In Section 2, the principal function formulation and path integral formulation of nonconservative systems for irregular Lagrangian are discussed. In Section 3, the definition of the wave function for irregular Lagrangian is explained. In Section 4, illustrative example is examined in detail. The work closes with some concluding remarks in Section 5.

\section{The Canonical Path Integral Formalism for Nonconservative Systems}

The Euler Lagrange equation for conservative systems is given by [17]

$$
\frac{\mathrm{d}}{\mathrm{d} t}\left(\frac{\partial L_{\circ}}{\partial \dot{q}}\right)-\frac{\partial L_{\circ}}{\partial q}=0
$$

In this work we would like to find the principal function for nonconservative systems using Euler Lagrange equation. We Start with the Lagrangian $L=L_{\circ}(q, \dot{q}) \mathrm{e}^{\lambda t}[18]$, where $L_{\circ}(q, \dot{q})$ stands for the Lagrangian of the corresponding conservative system. Because the Euler Lagrange equations are second order equations, we find the equations of motion from the corresponding Lagrangian in terms of the generalized coordinates and their derivatives. Then, we substitute the solutions of these equations in the given Lagrangian. Finally, the evolution of this Lagrangian between two instants of time $t_{1}$ and $t_{2}$ gives the principal function $S$ as a function of time.

$$
S(q, \dot{q}, t)=\int_{t_{1}}^{t_{2}} L_{\circ}(q, \dot{q}) \mathrm{e}^{\lambda t} \mathrm{~d} t=\int_{t_{1}}^{t_{2}} L(q, \dot{q}, t) \mathrm{d} t=\int_{t_{1}}^{t_{2}}(p \dot{q}-H) \mathrm{d} t
$$

where $H$ is the Hamiltonian function and $p$ is the canonical momentum.

The path integral representation by using Hamiltonian and Lagrangian mechanics may be written as

$$
\begin{aligned}
D(q, p) & =\int[\exp (i S)] \mathrm{d} p \mathrm{~d} q=\int\left[\exp i\left\{\int_{t_{1}}^{t_{2}}(p \dot{q}-H) \mathrm{d} t\right\}\right] \mathrm{d} p \mathrm{~d} q \\
& =\int\left[\exp i\left\{\int_{t_{1}}^{t_{2}} L(q, \dot{q}, t) \mathrm{d} t\right\}\right] \mathrm{d} p \mathrm{~d} q
\end{aligned}
$$

\section{Wave Function Definition for Irregular Lagrangian}

In the semi classical expansion (WKB) of the Hamilton Jacobi function of regular systems has been studied [11]. This expansion leads to the following wave function [19]:

$$
\psi\left(q_{i}, t\right)=\left[\prod_{i=1}^{N} \psi_{0 i}\left(q_{i}\right)\right]\left[\mathrm{e}^{\frac{i S(q, t)}{\hbar}}\right]
$$


where $\psi_{0 i}\left(q_{i}\right)$ is the amplitude of the wave function, which is defined as

$$
\psi_{0 i}\left(q_{i}\right)=\frac{1}{\sqrt{p\left(q_{i}\right)}} .
$$

We use the principal function to formulate the wave function.

\section{Example}

Let us consider one-dimensional Lagrangian of a free particle of mass $m$ in the presence of damping [15].

The Lagrangian is

$$
L=\frac{1}{2} m \dot{q}^{2} \mathrm{e}^{\lambda t}
$$

From Euler Lagrange equation

$$
\frac{\mathrm{d}}{\mathrm{d} t}\left(\frac{\partial L}{\partial \dot{q}}\right)-\frac{\partial L}{\partial q}=0
$$

The equation of motion is

$$
\ddot{q}+\lambda \dot{q}=0
$$

and the solution of Equation (8) is

$$
\dot{q}=C-\lambda q
$$

where $C$ is the constant of integration.

Integrating Equation (9) and choosing $q_{\circ}=0$ this gives

$$
q(t)=\frac{C}{\lambda}\left(1-\mathrm{e}^{-\lambda t}\right)
$$

Taking the first time derivative of Equation (10)

$$
\dot{q}=C \mathrm{e}^{-\lambda t}
$$

and

$$
\dot{q}^{2}=C^{2} \mathrm{e}^{-2 \lambda t}
$$

Substituting Equation (12) into Equation (6) we get

$$
L=\frac{1}{2} m C^{2} \mathrm{e}^{-\lambda t}
$$

Using Equation (2)

$$
S=\int_{0}^{t} \frac{1}{2} m C^{2} \mathrm{e}^{-\lambda t} \mathrm{~d} t
$$

Thus, the principal function takes the following form

$$
S=\frac{m C^{2}}{2 \lambda}\left[1-\mathrm{e}^{-\lambda t}\right]=\frac{m C}{2} q
$$

Also, the conjugate momentum is

$$
p=\frac{\partial S}{\partial q}=\frac{m C}{2}
$$


So that using Equations (15) and (16) the principal function can be written in the final form as following

$$
S(q, p)=p q
$$

Using Equation (5), we obtain the amplitude of the wave function as

$$
\psi_{0 i}\left(q_{i}\right)=\frac{1}{\sqrt{p\left(q_{i}\right)}}=\frac{1}{\sqrt{m C / 2}}
$$

Making use of Equation (4) the wave function can be written as

$$
\psi\left(q_{i}, t\right)=\psi_{0 i}\left(q_{i}\right) \mathrm{e}^{\frac{i S(q, t)}{\hbar}}=\frac{1}{\sqrt{m C / 2}} \mathrm{e}^{\frac{i m C q}{2 \hbar}}=\frac{1}{\sqrt{m C / 2}} \mathrm{e}^{\frac{i S(q, p)}{\hbar}}=\frac{1}{\sqrt{m C / 2}} \mathrm{e}^{\frac{i q p}{\hbar}}
$$

Finally, the path integral representation may be written as

$$
\begin{aligned}
D(q, p) & =\int[\exp (i S)] \mathrm{d} p \mathrm{~d} q=\int[\exp (i p q)] \mathrm{d} p \mathrm{~d} q \\
& =-i \ln |q|-i \sum_{n=1}^{\infty} \frac{(i p q)^{n}}{n \cdot n !}+A(q)+B(p)
\end{aligned}
$$

\section{Conclusion}

This paper is mainly concerned with path integral quantization of nonconservative systems. Nonconservative systems were studied within framework of Euler Lagrange equation. The corresponding complete integral, or principal function $S$, was determined using the method of time integral of the given Lagrangian. The momentum $p$ was calculated from $S$ in the usual manner. The appropriate wave function was then determined. The path integral for the nonconservative systems is obtained as an integration over the canonical phase space coordinates. One illustrative example is considered to demonstrate the application of our formalism.

\section{Conflicts of Interest}

The authors declare no conflicts of interest regarding the publication of this paper.

\section{References}

[1] Wiener, N. (1921) The Average of an Analytical Functional and the Brownian Movement. Proceedings of the National Academy of Sciences of the United States of America, 7, 294-298. https://doi.org/10.1073/pnas.7.10.294

[2] Dirac, P.A.M. (1933) The Lagrangian in Quantum Mechanics. Physikalische Zeitschrift der Sowjetunion, 3, 64-72.

[3] Feynman, R.P. (1948) Space-Time Approach to Non-Relativistic Quantum Mechanics. Reviews of Modern Physics, 20, 367-387. https://doi.org/10.1103/RevModPhys.20.367

[4] Rabei, E. and Guler, Y. (1992) Hamilton-Jacobi Treatment of Second-Class Constraints. Physical Review A, 46, 3513-3515.

https://www.ncbi.nlm.nih.gov/m/pubmed/9908518 
https://doi.org/10.1103/PhysRevA.46.3513

[5] Pimentel, B.M. and Teixeira, R.G. (1996) Hamilton-Jacobi Formulation for Singular Systems with Second-Order Lagrangians. Il Nuovo Cimento B, 111, 841-854.

https://doi.org/10.1007/BF02749015

[6] Pimentel, B.M. and Teixeira, R.G. (1998) Generalization of The Hamilton-Jacobi Approach for Higher-Order Singular Systems. Il Nuovo Cimento B, 113, 805-818. https://www.sif.it/riviste/sif/ncb/econtents/1998/113/06/article/1

[7] Muslih, S. and Guler, Y. (1997) The Feynman Path Integral Quantization of Constrained Systems. Il Nuovo Cimento B, 112, 97-107.

[8] Muslih, S. (2001) Path Integral Formulation of Constrained Systems with Singular-Higher Order Lagrangians. Hadronic Journal, 24, 713-720.

[9] Muslih, S.I. (2002) Quantization of Singular Systems with Second-Order Lagrangians. Modern Physics Letters A, 17, 2383-2391. https://doi.org/10.1142/S0217732302009027

[10] Rabei, E. (2000) On the Quantization of Constrained Systems Using Path Integral Techniques. Il Nuovo Cimento B, 115, 1159-1165. http://adsabs.harvard.edu/abs/2000NCimB.115.1159R

[11] Rabei, E., Nawafleh, K. and Ghassib, H. (2002) Quantization of Constrained Systems Using the WKB Approximation. Physical Review A, 66, Article ID: 024101. https://doi.org/10.1103/PhysRevA.66.024101

[12] Nawafleh, K., Rabei, E. and Ghassib, H. (2004) Hamilton-Jacobi Treatment of Constrained Systems. International Journal of Modern Physics A, 19, 347-354.

https://www.researchgate.net/.../228453409_Hamilton-Jacobi_Treatment_of_constr ained https://doi.org/10.1142/S0217751X04017719

[13] Hasan, E.H., Rabei, E.M. and Ghassib, H.B. (2004) Quantization of Higher-Order Constrained Lagrangian Systems Using the WKB Approximation. International Journal of Theoretical Physics, 43, 1073-1096. http://link.springer.com/article/10.1023/B:IJTP.0000049027.45011.37 https://doi.org/10.1023/B:IJTP.0000049027.45011.37

[14] Jarab'ah, O., Nawafleh, K. and Ghassib, H. (2013) Canonical Quantization of Dissipative Systems. European Scientific Journal, 9, 132-154.

[15] Hasan, E., Jarab'ah, O. and Nawafleh, K. (2014) Path Integral Quantization of Dissipative Systems. European Scientific Journal, 10, 308-314. http://www.eujournal.org/index.php/esj/article/download/3460/3223

[16] Jarab'ah, O. (2018) Path Integral Quantization of Regular Lagrangian. Applied Physics Research, 10, 9-13.

http://www.ccsenet.org/journal/index.php/apr/article/download/72127/40058 https://doi.org/10.5539/apr.v10n1p9

[17] Goldstein, H. (1980). Classical Mechanics. 2nd Edition, Addison-Wesley, Boston.

[18] Jarab'ah, O., Nawafleh, K. and Ghassib, H. (2013) A Hamilton-Jacobi Treatment of Dissipative Systems. European Scientific Journal, 9, 70-81. http://www.eujournal.org/index.php/esj/article/download/1946/1888

[19] Griffiths, D.J. (1995) Introduction to Quantum Mechanics. 2nd Edition, Prentice Hall, Upper Saddle River, New Jersey. 